\title{
Influence of elastic anisotropy on measured sound velocities and elastic moduli of polycrystalline cubic solids
}

\author{
Feng Xu, ${ }^{1, *}$ Philippe Djemia, ${ }^{2}$ Laurent Belliard,${ }^{3}$ Haijun Huang, ${ }^{1}$ Bernard Perrin ${ }^{3}$ and Andreas Zerr ${ }^{2}, *$ \\ ${ }^{1}$ School of Science, Wuhan University of Technology, 430070 Wuhan, China \\ ${ }^{2}$ Laboratoire des Sciences des Procédés et des Matériaux, CNRS UPR 3407, Université Sorbonne Paris \\ Nord, Alliance Sorbonne-Paris-Cité, 93430 Villetaneuse, France \\ ${ }^{3}$ Institut des NanoSciences de Paris, CNRS UMR 7588, Sorbonne Université, 75005 Paris, France
}

\begin{abstract}
Cubic solids such as $\mathrm{NaCl}$, crystalline argon, or $\mathrm{H}_{2} \mathrm{O}$-ice VII exhibit significant elastic anisotropy strongly increasing upon compression. As earlier recognized for solid argon and $\mathrm{H}_{2} \mathrm{O}$-ice (both exhibiting Zener ratio $A>1$ ), longitudinal sound velocities of their polycrystals, $V_{\mathrm{Lav}}$, measured using Brillouin light scattering (BLS) or pulse-echo ultrasonics are much closer to $V_{\mathrm{L}\langle 111\rangle}$ than to $V_{\mathrm{L}\langle 100\rangle}$, the $V_{\mathrm{L}}$-extremes in any cubic single crystal. Here, we experimentally confirm, using the technique of time-domain Brillouin scattering, the same tendency for $\mathrm{NaCl}$ exhibiting the opposite anisotropy type, $A<1$. To understand this tendency, we modelled orientational distribution and the frequency of occurrence of $V_{\mathrm{L}}$ values in texture-free polycrystalline samples of $\mathrm{NaCl}$ and solid argon. We found a remarkable and predictable asymmetry of the $V_{\mathrm{L}}$ distributions with maxima at $V_{\mathrm{L}\langle 110\rangle}$ which is always much closer to $V_{\mathrm{L}\langle 111\rangle}$. This asymmetry persists in BLS peaks but can be obscured in experiments. In the case of solid argon at $49 \mathrm{GPa}$, the asymmetry can lead to a moderate deviation of experimental $V_{\mathrm{Lav}}$ from $V_{\mathrm{LH}}$ (obtained from elastic-stiffness constants $C_{\mathrm{ij}}$ applying the Hill approximation) by $\sim 7 \%$. The latter can cause, however, a significant overestimation of the aggregate shear modulus by $\delta G / G \sim 50 \%$ or of the bulk modulus by $\delta B / B \sim 20 \%$ if just one BLS peak of longitudinal modes is detectable. A similar analysis, performed for transverse sound velocities, $V_{\mathrm{T}}$ and $V_{\mathrm{Tav}}$, has shown that, by use of a BLS spectrum showing peaks of both longitudinal and transverse modes, overestimation of $B$ is similarly high but that of $G$ is much less dramatic.
\end{abstract}

\section{Introduction}

Elastic anisotropy is a quantity describing non-uniformity of response of a single crystal to deformations along different directions, e.g., propagation of sound waves. While description of a single crystal under stress/deformation is straightforward, if the independent elastic-stiffness constants $C_{\mathrm{ij}}$ are known, description of response of a dense polycrystalline solid can be done only within of an approximation. The existing ones (e.g. Voigt, Reuss, Hill, Hashin-Shtrikman) were developed in order to take into account the influence of elastic anisotropy on distribution of strain and stress in adjacent grains of a deformed polycrystalline body. ${ }^{1-4}$ They involve assumptions about uniformity of strain and stress as well as of grain shapes and orientations. These approximations are used in various technical application such as engineering or building construction but are of exceptional importance for understanding of propagation of seismic waves in the deep Earth and, as a consequence, of its composition e.g.. ${ }^{5}$ According to earlier reports, a significant part of rock-forming minerals exhibits a moderate or decreasing with pressure elastic anisotropy. ${ }^{6,7}$ However, there are also cubic solids showing a strong increase of elastic anisotropy on compression, as recognized earlier ${ }^{8,9}$ and in the present work. For the latter type of cubic solids, a reliable recovery of elastic properties from experimental signals remains a subject of continuous efforts, e.g. when Brillouin light scattering (BLS) is applied to examine polycrystalline samples compressed in a diamond anvil cell (DAC). ${ }^{10}$

In the case of a cubic crystal, just three $C_{\mathrm{ij}}$ 's (namely, $C_{11}, C_{12}$ and $C_{44}$ ) are nonzero and the elastic anisotropy can be quantified using the Zener ratio $A=C_{44} / C^{\prime}$, the ratio of the two principal shear moduli, $C_{44}$ and $C^{\prime}=\left(C_{11}-C_{12}\right) / 2$. The first one, $C_{44}$, is related to simple shear along one of the symmetry directions while the second one, $C^{\prime}$, to the tetragonal shear. ${ }^{11}$ Cubic crystals can be classified with respect to the value of this Zener ratio, namely whether it

* Authors to whom correspondence should be addressed: xufeng@whut.edu.cn, zerr@univ-paris13.fr 
is greater or smaller than unity. As demonstrated below, orientationally isotropic polycrystals of these two classes of cubic solids show distinct signals by measurement of their averaged longitudinal and transverse sound velocity, $V_{\mathrm{Lav}}$ and $V_{\mathrm{Tav}}$, respectively. As a consequence, a careful signal analysis is required in order to avoid inconsistent $V_{\text {Lav }}$ and/or $V_{\text {Tav }}$ values as well as of bulk and/or shear moduli ( $B$ and/or $G$, respectively). Special attention should be paid not only to methods of measurement of $V_{\mathrm{Lav}}$ and/or $V_{\mathrm{Tav}}$, e.g. BLS or pulse-echo ultrasonics (PEUS), but also to procedures used to derive $B$ and $G$ from the collected signals taking into account grain sizes.

Until very recently, PEUS and BLS were the most common techniques used to measure $V_{\text {Lav }}$ and $V_{\text {Tav }}$ of densified polycrystalline solids. The latter technique is predominantly used to examine small/microscopic objects such as thin films or polycrystals compressed to ultrahigh pressures in a DAC. We show below, that shapes and widths of BLS peaks are controlled by elastic anisotropy, which can strongly increase with pressure and this in different ways for $A>1$ and $A<1$. Possibilities to experimentally analyze shapes of BLS peaks collected from microscopic samples, especially for transverse modes (T-modes) which peaks are much weaker than those of longitudinal modes (L-modes), are limited because it is difficult to compensate low signal-to-noise ratios by extending counting times: In some cases, measurement of only one BLS spectrum can last several days to just extract a peak from the background not to mention its shape. ${ }^{10,12}$ Apparently, $V_{\mathrm{Lav}}$ and $V_{\mathrm{Tav}}$ values recovered from such spectra require independent verification. Application of PEUS for measurement of $V_{\mathrm{Lav}}$ at ultrahigh pressures is, however, rare because its accuracy is strongly limited by sample sizes. Also, elastic anisotropy biases in a different way (depending on $A$ with respect to unity) $V_{\text {Lav }}$ and $V_{\text {Tav }}$ values recovered using the two techniques (see below).

In the last years, an alternative technique of time-domain Brillouin scattering (TDBS) was demonstrated to have significant advantages by examination of elastic behavior of microscopic polycrystalline samples due to its ability to measure longitudinal sound velocities with a high 3D-spatial resolution. This provides $V_{\mathrm{L}}$ variations for differently oriented crystallites or their groups and, as already described in earlier publications, access to the maximal and minimal $V_{\mathrm{L}}$ values, $V_{\mathrm{Lmax}}$ and $V_{\mathrm{Lmin}}$, along the fastest and the slowest directions in a single crystal. For cubic crystals, these are always $\langle 111\rangle$ and $\langle 100\rangle$, respectively, for $A>1$ and vice-versa for $A<1$. Combined with independently measured bulk modulus and density, knowledge of $V_{\mathrm{L}\langle 111\rangle}$ and $V_{\mathrm{L}\langle 100\rangle}$ provides access to the complete set of $C_{\mathrm{ij}}$ of any cubic solid. ${ }^{8}, 9$ The $V_{\text {Lav }}$ and $V_{\text {Tav }}$ values can then be derived using one of the approximations mentioned above.

In this work, we performed the first TDBS measurements on a polycrystalline sample of $\mathrm{NaCl}$ (having $A<1$ ) compressed to $24 \mathrm{GPa}$ in a DAC using the approach developed earlier. ${ }^{8,9,13-15}$ Our $V_{\mathrm{L}\langle 111\rangle}$ and $V_{\mathrm{L}\langle 100\rangle}$ data confirmed, within experimental uncertainties, the previous unique BLS data-point for a $\mathrm{NaCl}$ single crystal compressed to a similar pressure of $P=26 \mathrm{GPa},{ }^{10}$ as well as the experimental $C_{\mathrm{ij}}(P)$ derived from XRD examination of polycrystalline $\mathrm{NaCl}$ samples non-hydrostatically compressed in a DAC. ${ }^{16}$ Comparison of the existing and of our $V_{\mathrm{L}\langle 111\rangle}$ and $V_{\mathrm{L}\langle 100\rangle}$ values with earlier experimental dependences $V_{\mathrm{Lav}}(P)$ for $\mathrm{NaCl}$ polycrystals revealed a significant shift of the latter towards $V_{\mathrm{L}\langle 111\rangle}(P)$. In order to understand nature of this shift, recognized earlier for anisotropic cubic solids with $A>1$ where $V_{\mathrm{Lav}}(P)$ was also found to approach $V_{\mathrm{L}\langle 111\rangle}(P),{ }^{8,9}$ we performed a detailed analysis of possible origins. For this aim, we modelled shapes of BLS peaks of strongly anisotropic polycrystalline cubic solids with $A<1$ $(\mathrm{NaCl})$ and $A>1$ (solid argon). We have found a remarkable but predictable asymmetry of the peak shapes which make determining of both $V_{\mathrm{Lav}}$ and $V_{\text {Tav }}$ and of the isotropic elastic moduli $B$ and $G$ ambiguous. We show that ambiguities in determining of $V_{\mathrm{Lav}}$ and $V_{\mathrm{Tav}}$ are different for the two classes of elastically anisotropic cubic solids $(A<1$ or $A>1)$ and for the classical measuring techniques, BLS and PEUS, especially when an operator is involved in the decision about positions of the detected BLS peaks. However, some systematics have also been recognized.

\section{TDBS measurements of $C_{\mathrm{ij}}$ of $\mathrm{NaCl}$ at $24 \mathrm{GPa}$}

In our experiment, $\mathrm{NaCl}$ powder was compressed in a $\mathrm{DAC}$ between beveled diamond anvils and radially supported by a pre-indented steel gasket. A thin metallic film $(\mathrm{Ti}, 350 \mathrm{~nm}$ in thickness), facing the sample on one of the sides, served as a photo-acoustic transducer which absorbed ultrashort pulses of a pump laser and lunched coherent acoustic pulses (CAPs) into 
the sample (Fig. 1). ${ }^{13-15}$ Ruby grains placed near the transducer were used for pressure measurement. 3D-spatially resolved velocity of propagation of the CAPs along the DAC axis was measured using a probe laser which pulses could be continuously delayed in time with respect to the pump-laser pulses: The probe laser light, scattered by a moving CAP, interfered on a photodetector with that reflected from stationary optical inhomogeneities and thus produced a signal oscillating as a function of the delay-time between the pump- and probe pulses $(\Delta t)$ and, accordingly, as a function of the distance from the transducer to the CAP. ${ }^{13-15,17}$ Figure 1a shows one of raw TDBS signals collected in our experiments which shows oscillations due to the propagation of a CAP (named Brillouin oscillations ${ }^{18-20}$ ) superimposed with non-oscillating background, caused by a transient dissipation of heat from the sample area hit by the pump laser pulse. Frequencies of the oscillations obey the same equation as in classical BLS measurements: $f_{\mathrm{B}}=2 n V_{\mathrm{L}} / \lambda$, where $f_{\mathrm{B}}$ is the Brillouin frequency, $\lambda$ wavelength of the probe light in vacuum and $n$ refractive index of the sample material. Accordingly, CAPs propagating through differently oriented elastically anisotropic crystallites generate oscillations of the scattered probe light with $f_{\mathrm{B}}$ proportional to the local $V_{\mathrm{L}}$ (in the crystallites or their groups) along the CAP propagation direction (parallel to the sample axis). ${ }^{14,15}$ In order to axially resolve the $V_{\mathrm{L}}$ values, the short-time Fourier transform (STFT) was applied with a narrow temporal window $\Delta t$ equal to one oscillation which defined the axial/depth resolution to be $\sim 250 \mathrm{~nm}$. Our measurements have been performed on three different samples compressed to $P=24(1) \mathrm{GPa}$ and, for each sample, TDBS signals were collected from typically 2 different places. Considering that each TDBS signal provided up to two dozens of independent $f_{\mathrm{B}}$ values (see Brillouin oscillations in Fig. 1), more than 100 independent locally resolved $V_{\mathrm{L}}$ values were measured for differently oriented grains or group of grains. To derive $V_{\mathrm{L}}$ values from the Brillouin frequencies we used here the refractive index $n=1.698$ from our ab-initio calculations (to be reported separately ${ }^{21}$ ). From all the $V_{\mathrm{L}}$ values we extracted the maximal and the minimal ones, $V_{\mathrm{Lmax}}$ and $V_{\mathrm{Lmin}}$, respectively. For cubic solids, the latter pair represents, with a high degree of confidence, $V_{\mathrm{L}\langle 111\rangle}$ and $V_{\mathrm{L}\langle 100\rangle}$ or vice-versa, even though the sample is textured.

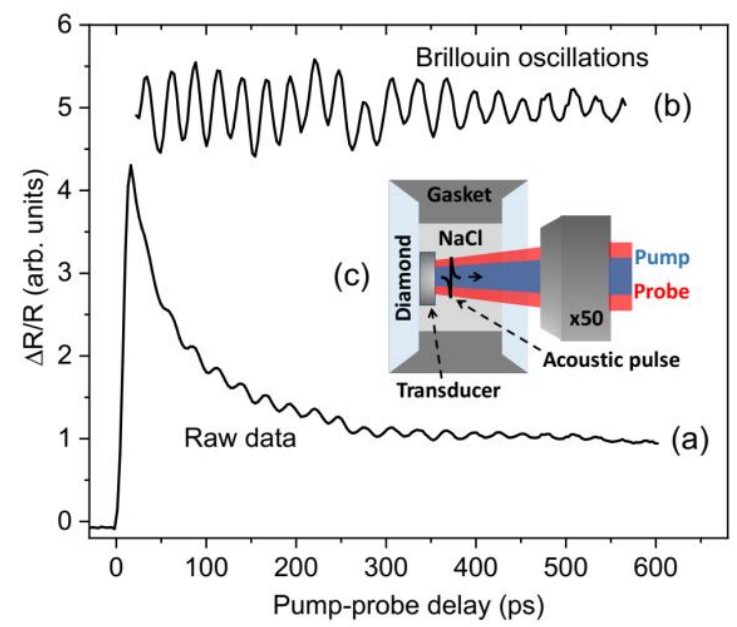

FIG. 1. (a) TDBS raw signal: relative change of transient reflectivity, as a function of the pump-probe delay, recorded for a polycrystalline $\mathrm{NaCl}$ sample compressed to 24(1) GPa. (b) Brillouin oscillations after subtraction of the thermal background from the raw TDBS signal shown in (a). Amplitude of the oscillations was magnified for better visibility. (c) Schematic of the sample inside the DAC and of paths of the pump and probe laser beams.

The $V_{\mathrm{Lmax}}$ and $V_{\mathrm{Lmin}}$ extracted here for $\mathrm{NaCl}$ correspond, respectively, to $V_{\mathrm{L}\langle 100\rangle}$ and $V_{\mathrm{L}\langle 111\rangle}$ (Fig. 2). Using the obtained $V_{\mathrm{L}\langle 100\rangle}=9.0(6) \mathrm{km} / \mathrm{s}$ and $V_{\mathrm{L}\langle 111\rangle}=7.0(3) \mathrm{km} / \mathrm{s}$, we recovered all single-crystal elastic moduli $C_{11}=266(24) \mathrm{GPa}, C_{12}=49(12) \mathrm{GPa}$ and $C_{44}=28(11) \mathrm{GPa}$ applying $B(P)=119.5 \mathrm{GPa}$ from the Decker's equation of state, $\rho(P) .{ }^{22}$ Then we calculated $V_{\mathrm{L}\langle 110}=7.6(3)$ $\mathrm{km} / \mathrm{s}$ (Fig. 2), shear modulus $G_{\mathrm{H}}=50$ (13) $\mathrm{GPa}$ and the anisotropy ratio $A=0.27(10)$. To do this, we used the following equations ${ }^{23}$ : 


$$
\begin{gathered}
V_{\mathrm{L}\langle 100\rangle}=\sqrt{\frac{C_{11}}{\rho}} ; V_{\mathrm{L}\langle 110\rangle}=\sqrt{\frac{C_{11}+C_{12}+2 C_{44}}{2 \rho}} ; V_{\mathrm{L}\langle 111\rangle}=\sqrt{\frac{C_{11}+2 C_{12}+4 C_{44}}{3 \rho}}=\sqrt{\frac{B}{\rho}+\frac{4 C_{44}}{3 \rho}} \\
B=\frac{C_{11}+2 C_{12}}{3} ; G_{H}=\frac{1}{2}\left(G_{V}+G_{R}\right)=\frac{1}{2}\left(\frac{C_{11}-C_{12}+3 C_{44}}{5}+\frac{5\left(C_{11}-C_{12}\right) C_{44}}{4 C_{44}+3\left(C_{11}-C_{12}\right)}\right)
\end{gathered}
$$

where $G_{\mathrm{V}}, G_{\mathrm{R}}$ and $G_{\mathrm{H}}$ represent shear moduli according to the Voigt-, Reuss- and Hill approximation, respectively. Our experimental $V_{\mathrm{L}\langle 100\rangle}$ and $V_{\mathrm{L}\langle 111\rangle}$ of $\mathrm{NaCl}$ at $P=24 \mathrm{GPa}$ agree, within the experimental errors, with results of the unique classical BLS measurement on a single crystal of $\mathrm{NaCl}$ at a similarly high pressure of $P=26 \mathrm{GPa}^{10}$ (Fig. 2). They also agree reasonably well with results of a rarely used approach based on radial-XRD measurements on powders compressed in a $\mathrm{DAC}^{16}$ (Fig. 2). In the latter approach, nonhydrostatic compression causes a systematic difference in the interplanar distances $d(h k l)$ due to the presence of uniaxial stress which permits to derive, making some assumptions, all $C_{\mathrm{ij}}(P)$ of cubic solids. Earlier reported $V_{\text {Lav }}$ values of polycrystalline $\mathrm{NaCl}$, measured using the conventional BLSand PEUS techniques, are located between our $V_{\mathrm{L}\langle 100\rangle}$ and $V_{\mathrm{L}\langle 111\rangle}$ and show the tendency to approach $V_{\mathrm{L}\langle 111\rangle}$ (Fig. 2).

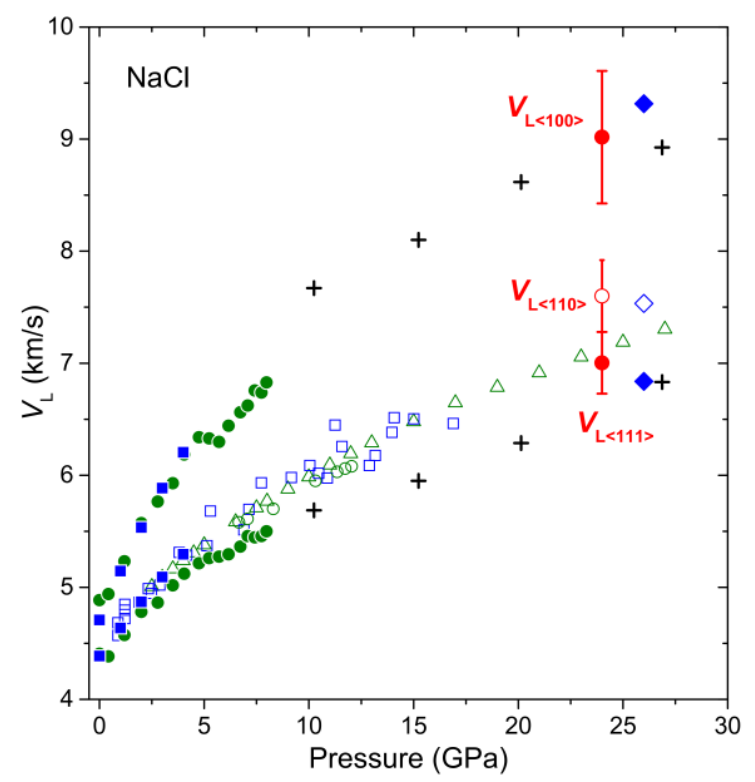

FIG. 2. Pressure dependences of longitudinal sound velocities of $\mathrm{NaCl}$ measured using different methods. The $V_{\mathrm{L}\langle 100\rangle}$ and $V_{\mathrm{L}\langle 111\rangle}$ derived from our TDBS measurements on a polycrystalline sample of $\mathrm{NaCl}$ at $P=24 \mathrm{GPa}$ are shown by solid red circles with error bars. They agree with the earlier measurements on $\mathrm{NaCl}$ single crystals using classical BLS (solid blue diamonds ${ }^{10}$ and solid blue square ${ }^{24}$ ) and pulse-echo ultrasonics (solid green circles ${ }^{25}$ ) as well as with $V_{\mathrm{L}\langle 100\rangle}$ and $V_{\mathrm{L}\langle 111\rangle}$ derived from the radial-XRD examination of $\mathrm{NaCl}$ polycrystals compressed nonhydrostatically (crosses $\left.{ }^{16}\right)$. The open red circle with error bars indicates $V_{\mathrm{L}\langle 110\rangle}$ calculated from our $V_{\mathrm{L}\langle 100\rangle}$ and $V_{\mathrm{L}\langle 111\rangle}$ (see Eq. (3) below) while the open blue diamond shows $V_{\mathrm{L}\langle 110\rangle}$ from the classical BLS measurement at $26 \mathrm{GPa} .{ }^{10} \mathrm{The}$ earlier experimental $V_{\mathrm{Lav}}(P)$ for polycrystalline $\mathrm{NaCl}$ are shown as follows: classical BLS results - open blue squares ${ }^{26}$; ultrasonic measurements - open green triangle ${ }^{27}$ and open green circles. ${ }^{28}$

\section{III. $V_{\mathrm{L}}$ distribution in elastically anisotropic cubic crystals}

The fact that $V_{\text {Lav }}$ obtained in classical BLS measurements and, in particular cases considered below, in PEUS measurements are much closer to $V_{\mathrm{L}\langle 111\rangle}$ than to $V_{\mathrm{L}\langle 100\rangle}$ was first recognized in high-pressure experiments on $\mathrm{H}_{2} \mathrm{O}$ ice ${ }^{8}$ and argon ${ }^{9}$ crystallizing on compression in cubic structures. The important difference of these two solids from $\mathrm{NaCl}$ is the inverse ratio of the velocity extremes: In $\mathrm{H}_{2} \mathrm{O}$ ice and cubic argon, $V_{\mathrm{L}\langle 111\rangle}$ is not the slowest but the fastest direction of sound propagation in a single crystal. In the present and in the previous works, first application of high-pressures permitted to reveal this particular tendency for $V_{\text {Lav }}$ because 
elastic anisotropy of these compounds grew on compression to the levels where the tendency surpassed experimental uncertainties.

In order to understand this tendency, we performed a detailed analysis of distribution of sound velocities in $\mathrm{NaCl}$ (at $24 \mathrm{GPa}$ and $1 \mathrm{~atm}$.) and in solid argon (at $49 \mathrm{GPa}$ and $2.4 \mathrm{GPa}$ ) which were selected as the representatives of cubic solids with $A<1$ and $A>1$, respectively. The pressure of $24 \mathrm{GPa}$ was chosen for $\mathrm{NaCl}$ because its elastic anisotropy approached the maximum but the transition to the high-pressure B2 phase at $\sim 30 \mathrm{GPa}$ not yet commenced. ${ }^{29}$ The reason for choosing $P=49 \mathrm{GPa}$ for solid argon is the same: at this pressure its elastic anisotropy approaches the highest measured value. ${ }^{9}$ The pressure of $P=2.4 \mathrm{GPa}$ is just above the value where condensed argon solidifies. ${ }^{30}$ Another reason for the above selections is a similarly high value of $A$, in the case of solid argon, and of its inverse, $1 / A$, in the case of $\mathrm{NaCl}$.

At the first step, we calculated $V_{\mathrm{L}}$-histograms representing the probability to measure each of the possible $V_{\mathrm{L}}$ 's along a fixed direction in space when a single crystal of $\mathrm{NaCl}$ (Fig. 3a-b) or of solid argon (Fig. 3d-e) is uniformly rotated in the 3D space. Such a histogram is equivalent to statistical distribution of $V_{\mathrm{L}}$ in a texture-free polycrystalline sample and, accordingly, to the ideal shape of its BLS peak collected for a particular scattering direction if the instrument function, background and noise of the experimental set-up are neglected. This statement is valid when wavelengths of longitudinal waves, $\lambda_{\mathrm{ac}}$, scattering the incident laser radiation are shorter than typical size of crystallites constituting the sample. The latter is usually the case in classical BLS measurements on common polycrystalline samples free of intentionally added $\mathrm{nm}$-sized grains (typically $<100 \mathrm{~nm}$ ) which elastic properties can, in addition, deviate from those of sub- $\mu \mathrm{m}$ - and $\mu \mathrm{m}$-sized grains in common polycrystalline solids. More precisely, such histograms can be attributed to BLS peaks collected in the backscattering geometry, $\theta=180^{\circ}$, if the examined polycrystalline $\mathrm{NaCl}$ sample is composed of grains larger than $\sim 160 \mathrm{~nm}$. The latter value is similar to or above the wavelength $\lambda_{\mathrm{ac}}=\lambda_{\mathrm{L}} /(2 n \sin (\theta / 2))$ of acoustic waves probed using a typical laser with the wavelength of $\lambda_{\mathrm{L}}=532 \mathrm{~nm}$. In the case of the $\theta=90^{\circ}$ scattering geometry, the grain-size limitation increases to $\sim 250 \mathrm{~nm}$, which is still small.

Figures $3 \mathrm{a}$ and $3 \mathrm{~b}$ show $V_{\mathrm{L}}$-histograms calculated for $\mathrm{NaCl}$ using $C_{\mathrm{ij}}$ measured for a single crystal at $P=1 \mathrm{~atm}^{25}$ and those measured here at $P=24 \mathrm{GPa}$, respectively. Figures $3 \mathrm{~d}$ and $3 \mathrm{e}$ show histograms calculated for solid argon using earlier reported experimental $C_{\mathrm{ij}}$ at $P=2.4$ $\mathrm{GPa}^{30}$ and at $P=49 \mathrm{GPa},{ }^{9}$ respectively. For both solids, it is the increase of anisotropy on compression which causes broadening of the histograms over significant $V_{\mathrm{L}}$-ranges and, more importantly, their asymmetric shapes with sharp maxima: For $\mathrm{NaCl}$ at $P=1 \mathrm{~atm}$. and $P=24$ GPa, the peak maximum is located at $V_{\mathrm{L}}=4.5 \mathrm{~km} / \mathrm{s}$ and $V_{\mathrm{L}}=7.6 \mathrm{~km} / \mathrm{s}$, respectively, closer to the lower end of the histograms limited by $V_{\mathrm{L}\langle 111\rangle}$ (Figures $3 \mathrm{a}$ and $3 \mathrm{~b}$ ). For solid argon at $P=2.4$ GPa and $P=49 \mathrm{GPa}$, the peak maximum is located at $V_{\mathrm{L}}=3.3 \mathrm{~km} / \mathrm{s}$ and $V_{\mathrm{L}}=8.2 \mathrm{~km} / \mathrm{s}$, respectively, closer to the upper end of the histograms limited, again, by $V_{\mathrm{L}\langle 111\rangle}$ (Figures $3 \mathrm{~d}$ and 3e). Maxima of these peaks correspond, for each of the solids, to $V_{\mathrm{L}\langle 110\rangle}$ exactly, as followed from our direct calculations of $V_{\mathrm{L}\langle 110\rangle}$ using the same experimental $C_{\mathrm{ij}}$ 's (Fig. 3). Appearance of such clear local maxima in the histograms is due to the fact that (i) orientations along and close to $\langle 110\rangle$ are highly populated in cubic crystals and (ii) variation of $V_{\mathrm{L}}$ with orientation is monotonous. The latter can be recognized from 3D representations of orientational distribution of $V_{\mathrm{L}}$ in a single crystal of $\mathrm{NaCl}$ at $24 \mathrm{GPa}$ (Fig. 3c), and in a single crystal of solid argon at $49 \mathrm{GPa}$ (Fig. 3f). For both solids, the distribution of $V_{\mathrm{L}}$ is of the same character except the directions corresponding to the maximal and minimal $V_{\mathrm{L}}$ are inversed. Moreover, the position of $V_{\mathrm{L}\langle 110\rangle}$ between $V_{\mathrm{L}\langle 100\rangle}$ and $V_{\mathrm{L}\langle 111\rangle}$ is not arbitrary but defined by the equation:

$$
V_{\mathrm{L}\langle 100\rangle}^{2}-V_{\mathrm{L}\langle 110\rangle}^{2}=3\left(V_{\mathrm{L}\langle 110\rangle}^{2}-V_{\mathrm{L}\langle 111\rangle}^{2}\right)
$$

which can be easily verified by substitution of squares of $V_{\mathrm{L}\langle\mathrm{hkl}\rangle}$ (given in Eqs. (1)) in the present Eq. (3). In the case of a weak elastic anisotropy, when $A$ approaches unity, $\left|V_{\mathrm{L}\langle 111\rangle}-V_{\mathrm{L}\langle 100\rangle}\right|<<V_{\mathrm{L}\langle\text { hkl }\rangle}$ (for any $h, k$ and/or $\left.l\right)$ and $\left(V_{\mathrm{L}\langle 100\rangle}+V_{\mathrm{L}\langle 110\rangle)}\right) /\left(V_{\mathrm{L}\langle 110\rangle}+V_{\mathrm{L}\langle 111\rangle}\right)$ is close to unity. Then Eq. (3), rewritten as:

$$
\left(V_{\mathrm{L}\langle 100\rangle}-V_{\mathrm{L}\langle 110\rangle}\right)\left(V_{\mathrm{L}\langle 100\rangle}+V_{\mathrm{L}\langle 110\rangle}\right)=3\left(V_{\mathrm{L}\langle 110\rangle}-V_{\mathrm{L}\langle 111\rangle}\right)\left(V_{\mathrm{L}\langle 110\rangle}+V_{\mathrm{L}\langle 111\rangle}\right)
$$

can be simplified to: 


$$
V_{\mathrm{L}\langle 100\rangle}-V_{\mathrm{L}\langle 110\rangle} \approx 3\left(V_{\mathrm{L}\langle 110\rangle}-V_{\mathrm{L}\langle 111\rangle}\right)
$$

The latter result indicates that the difference $\left|V_{\mathrm{L}\langle 110\rangle}-V_{\mathrm{L}\langle 111\rangle}\right\rangle$ makes only $25 \%$ of the maximal possible spreading of $V_{\mathrm{L}}$ 's in a cubic single crystal, equal to $\left|V_{\mathrm{L}\langle 100\rangle}-V_{\mathrm{L}\langle 111\rangle}\right\rangle$.

Further, we calculated $V_{\text {Lav }}$ values applying the Voigt-, Reuss-, and Hill approximations ( $V_{\mathrm{LV}}$, $V_{\mathrm{LR}}$, and $V_{\mathrm{LH}}$, respectively) using the same $C_{\mathrm{ij}}$ as by establishing of the histograms and compared them with $V_{\mathrm{L}\langle 110\rangle}$ in Fig. 3a-b and 3d-e. Here, $V_{\mathrm{LH}}$ can be attributed to sound velocity in an isotropic polycrystalline solid when $\lambda_{\mathrm{ac}}$ is greater than grain sizes. The latter is usual in PEUS measurements and could be the case in particular BLS measurements with small scattering angles $\theta$ when polycrystals contain grains of intermediate sizes, between about 100 and $250 \mathrm{~nm}$. We also derived the value $V_{\mathrm{LC}}$ indicating center of mass of the histogram which can be attributed to $V_{\text {Lav }}$ in rare PEUS measurements where $\lambda_{\text {ac }}$ is smaller than grain sizes. Common for such case is a higher value of $V_{\mathrm{LC}}$ when compared with $V_{\mathrm{LH}}$, independent of $A$ (see Fig. 3a, b, d, e).

Applying the Voigt approximation to estimate sound velocity in an isotropic polycrystalline solid:

$$
V_{\mathrm{LV}}=\sqrt{\frac{9 C_{11}+6 C_{12}+12 C_{44}}{15 \rho}}
$$

and Eq. (1) for $V_{\mathrm{L}\langle 110\rangle}$, we found, in addition, that:

$$
V_{\mathrm{L}\langle 110\rangle}^{2}-V_{\mathrm{LV}}^{2}=\frac{(A-1) C^{\prime}}{5 \rho}
$$

This indicates that, for all cubic solids with $A>1$ (such as argon), $V_{\mathrm{L}\langle 110\rangle}$ is always higher than $V_{\mathrm{LV}}$ and, consequently, than $V_{\mathrm{LH}}$ and $V_{\mathrm{LR}}$ (Fig. 3d-e). Accordingly, maxima of ideal BLS peaks, if selected by an operator, will provide overestimated $V_{\mathrm{Lav}}$ (similar to $V_{\mathrm{L}\langle 110\rangle}$ ) when compared with $V_{\mathrm{LH}}$ from, for example, PEUS measurements. In the case of solids with $A<1$ (e.g. $\mathrm{NaCl}), V_{\mathrm{L}\langle 110\rangle}$ is always smaller than $V_{\mathrm{LV}}$ but $V_{\mathrm{LR}}$ can be either smaller or greater than $V_{\mathrm{L}\langle 110\rangle}$ but $V_{\mathrm{LH}}$ appears to be always similar to $V_{\mathrm{L}\langle 110\rangle}(\mathrm{Fig}$. 3a, b).

Another interesting observation valid for both cubic solids considered here, e.g. for $A>1$ and for $A<1$, is an apparent growth with pressure of the difference between the approximations of velocity in polycrystalline solids $V_{\mathrm{LR}}, V_{\mathrm{LH}}$ and $V_{\mathrm{LV}}$, on one hand, and $V_{\mathrm{LC}}$, on the other. In order to verify this statement, we evaluated the distance between $V_{\mathrm{LV}}$ (representing the group $V_{\mathrm{LR}}, V_{\mathrm{LH}}$ and $\left.V_{\mathrm{LV}}\right)$ and $V_{\mathrm{Lmax}}$ representing $V_{\mathrm{LC}}$. The latter replacement, needed because $V_{\mathrm{LC}}$ cannot be expressed analytically, is plausible because, for each of the considered two solids, $V_{\mathrm{L}}$ 's redistribute towards the histogram part with higher velocities (see Fig. 3b, e) and $V_{\mathrm{LC}}$ moves, accordingly, towards $V_{\mathrm{Lmax}}$. For $\mathrm{NaCl}$ having $A<1, V_{\mathrm{L}\langle 100\rangle}$ is the upper extreme (Fig. 3b) and the distance to $V_{\mathrm{LV}}$ changes according to the relation:

$$
V_{\mathrm{L}\langle 100\rangle}^{2}-V_{\mathrm{LV}}^{2}=\frac{4(1-A) C^{\prime}}{5 \rho}
$$

From $1 \mathrm{~atm}$. to $24 \mathrm{GPa}$, this difference increases with pressure because (1-A) increases by 2 times (Fig. 3a and 3b) and the basic shear modulus $C^{\prime}$ increases also. On the other hand, the denominator $\rho$ increases in this pressure range by $12 \%$ only. ${ }^{22}$ In the case of solid argon having $A>1, V_{\mathrm{L}\langle 111\rangle}$ is the upper extreme and the distance to $V_{\mathrm{LV}}$ changes according to the equation:

$$
V_{\mathrm{L}\langle 111\rangle}^{2}-V_{\mathrm{LV}}^{2}=\frac{8(A-1) C^{\prime}}{15 \rho}
$$

From 2.4 GPa to $49 \mathrm{GPa}$, this difference increases too because $A$ increases from $\sim 3$ to $\sim 18$ and $(A-1)$, accordingly, by more than eight times while $C^{\prime}$ also grows. The denominator $\rho$ increases, in the same pressure range, by two times only. ${ }^{31}$ Consequently, the difference between $V_{\mathrm{LV}}$ (representing the group $V_{\mathrm{LR}}, V_{\mathrm{LH}}$ and $V_{\mathrm{LV}}$ ) and $V_{\mathrm{Lmax}}$ (representing $V_{\mathrm{LC}}$ ) increases with pressure for both $A<1$ and $A>1$ if pressure dependence of elastic anisotropy is stronger than that of the ratio $\rho / C^{\prime}$. 

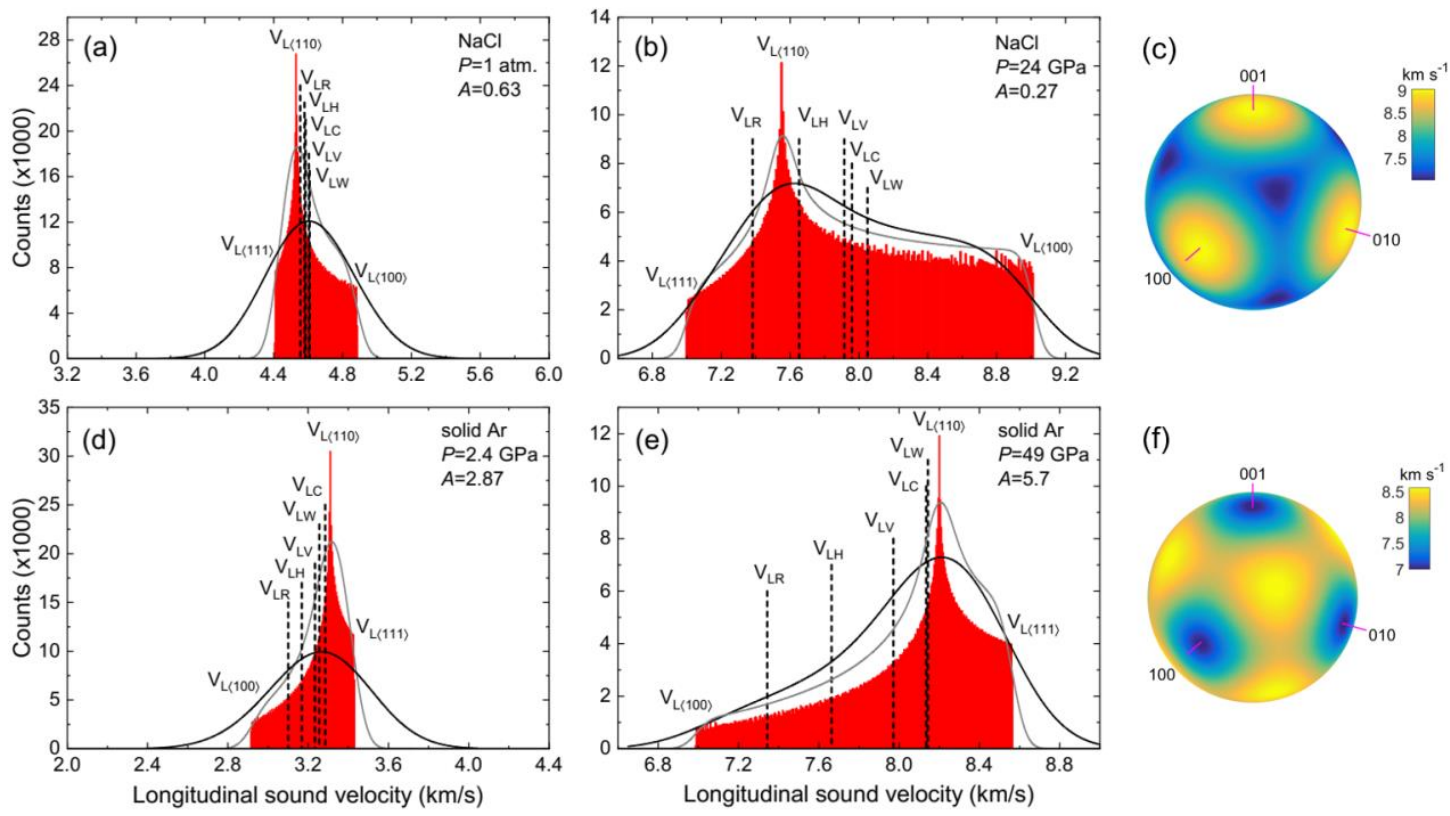

FIG. 3. Histograms of the frequency of occurrence of $V_{\mathrm{L}}$ values in a cubic single crystal of $\mathrm{NaCl}$ (a and b) and of solid argon (d and e), and 3D representations of $V_{\mathrm{L}}$ values (color scales) along different directions in single crystals of $\mathrm{NaCl}$ (c) and of solid argon (f). The histograms are equivalent to statistical distributions of $V_{\mathrm{L}}$ in texture-free polycrystalline samples measured, at the indicated pressure, for a selected sound propagation direction. The histograms for $\mathrm{NaCl}$ (red bars) were calculated using the earlier reported experimental $C_{\mathrm{ij}}$ at $1 \mathrm{~atm}^{25}$ (a) and our experimental $C_{\mathrm{ij}}$ at $24 \mathrm{GPa}$ (b). The histograms for solid argon (red bars) were calculated using earlier published experimental $C_{\mathrm{ij}}$ at 2.4 $\mathrm{GPa}^{30}$ (d) and $49 \mathrm{GPa}^{9}(\mathrm{e}) . V_{\mathrm{L}\langle 100\rangle}$ and $V_{\mathrm{L}\langle 111\rangle}$ are the maximal and the minimal $V_{\mathrm{L}}$ values for $\mathrm{NaCl}$ having $A<1$, while the case is opposite for solid argon having $A>1$. Vertical dashed lines in (a, b, d, e) indicate the velocities $V_{\mathrm{LV}}, V_{\mathrm{LR}}$ and $V_{\mathrm{LH}}$ obtained using the three well-known approximations, Voigt, Reuss, and Hill, respectively. Solid curves represent modelled shapes of BLS-peaks for isotropic densified polycrystalline samples obtained via convolution of the histograms with Gaussian distributions having widths of $0.25 \mathrm{GHz}$ (gray lines) and of $1 \mathrm{GHz}$ (black lines). The vertical dashed lines labeled with $V_{\mathrm{LC}}$ indicate positions of mass-centers of the histograms while $V_{\mathrm{LW}}$ indicate positions of FWHM-middles of the BLS peaks modelled using the wider Gaussian distribution.

As mentioned above, the histograms shown in Fig. 3 can be considered as ideal representations of intensity distribution in BLS peaks collected from polycrystalline texture-free samples if grains composing them are sufficiently large (above 160-250 nm, depending on the BLS geometry), background, noise and all line-broadening effects are negligible. In order to take line-broadening into account, we modelled shapes of experimental BLS-peaks by convolution of the histograms with Gaussian distributions having widths of $0.25 \mathrm{GHz}$ and of $1 \mathrm{GHz}$. The latter value was taken from a BLS spectrum collected from a $\mathrm{NaCl}$ single-crystal compressed to $P=26 \mathrm{GPa}$ (see figure 10 in Ref. 6). It appears to be mostly caused by a significant numerical aperture of the optics used to collect the scattered light because it exceeds the instrument function of a typical BLS spectrometer. The latter we estimated to be $\sim 0.25 \mathrm{GHz}$ or $\sim 1 \%$ of the range of frequencies (proportional to $V_{\mathrm{L}}$ ) covered by the spectrum in the same figure 10 in Ref. 6. As shown in Fig. 3, the peak shapes modelled using the narrower Gaussian distribution resemble shapes of the histograms. However, the peak models obtained using the wider Gaussian distribution obscure the histogram asymmetry, especially for the weaker anisotropy. Apparently, the BLS peak asymmetry will further be obscured by electronic noise, background, not to mention texture in the examined polycrystalline sample. If texture is not pronounced then middle of the full width at half maximum (FWHM) of the recorded BLS-peak, $V_{\mathrm{LW}}$, is usually attributed to $V_{\mathrm{Lav}}$. In the particular cases of $\mathrm{NaCl}$ compressed to $P=24 \mathrm{GPa}$ and of solid argon compressed to $P=49$ $\mathrm{GPa}, V_{\mathrm{LW}}$ values are $\sim 5-7 \%$ higher when compared with the corresponding $V_{\mathrm{LH}}$ values (Fig. $3 \mathrm{~b}$ and $3 \mathrm{e}$, respectively). In general, the extracted $V_{\mathrm{Lav}}$ will depend, for such signals, on the 
method used to determine the BLS-peak position. These could be the peak maximum, its center-of-mass, or middle of the FWHM given by $V_{\mathrm{L}\langle 110\rangle}, V_{\mathrm{LC}}$ or $V_{\mathrm{LW}}$, respectively. Agreement of $V_{\text {Lav }}(P)$ of polycrystalline $\mathrm{NaCl}$ from PEUS measurements ${ }^{27}$ with that from the BLS measurements ${ }^{26}$ suggest that BLS-peak maxima and not $V_{\mathrm{LW}}$ were preferred in the latter work. However, we cannot exclude that texturing of the $\mathrm{NaCl}$ samples upon compression between opposed anvils even stronger promoted the mutual agreement and the tendency of $V_{\mathrm{Lav}}$ to approach $V_{\mathrm{L}\langle 111\rangle}$ (Fig. 2).

In the case of solids having $A>1$, such as argon, the values of $V_{\mathrm{LC}}, V_{\mathrm{LW}}$ and $V_{\mathrm{L}\langle 110\rangle}$ are similar and the derived $V_{\text {Lav }}$ will not depend on the BLS-peak quality and the operator choice (Fig. $3 \mathrm{e})$. On the other hand, all established approximations of sound velocities provide lower values of $V_{\mathrm{LV}}, V_{\mathrm{LH}}$ and $V_{\mathrm{LR}}$. Accordingly, $V_{\mathrm{Lav}}=V_{\mathrm{LH}}$ obtained using PEUS will disagree with the BLS result, where $V_{\mathrm{Lw}}$ should be selected. Thus, BLS measurements on polycrystals with $A>1$ are expected to overestimate $V_{\mathrm{Lav}}$ and cause the tendency of approaching of $V_{\mathrm{L}\langle 111\rangle}$ by $V_{\text {Lav. }}$.

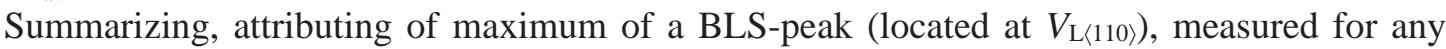
polycrystalline sample of an elastically anisotropic cubic solid, to $V_{\mathrm{Lav}}$ will always lead to the recognized approaching of $V_{\mathrm{L}\langle 111\rangle}$ by $V_{\mathrm{Lav}}$. For solids with $A<1, V_{\mathrm{Lav}}$ attributed to $V_{\mathrm{L}\langle 110\rangle}$ would agree with $V_{\mathrm{LH}}$ and thus with PEUS data. For solids with $A>1, V_{\mathrm{Lav}}$ attributed to $V_{\mathrm{L}\langle 110\rangle}$ would be systematically higher than $V_{\mathrm{LH}}$. The case where $V_{\mathrm{Lav}}$ is attributed to $V_{\mathrm{LW}}$ is less straightforward: For solids with $A<1$, such $V_{\mathrm{Lav}}=V_{\mathrm{LW}}$ will deviate from $V_{\mathrm{LH}}$ and provide overestimated values. For solids with $A>1$, such $V_{\mathrm{Lav}}=V_{\mathrm{LW}}$ will provide a value similar to $V_{\mathrm{L}\langle 110\rangle}$, thus been closer to $V_{\mathrm{L}\langle 111\rangle}$ than to $V_{\mathrm{L}\langle 100\rangle}$ and, again, higher than $V_{\mathrm{LH}}$. With other words, $V_{\text {Lav }}$ recovered from BLS measurements on polycrystalline elastically anisotropic cubic samples using any of the approaches will be either equal to or higher than $V_{\mathrm{LH}}$, independent of the Zener ratio $A$.

\section{IV. $V_{\mathrm{T}}$ distribution in elastically anisotropic cubic crystals}

In this section, we examine theoretically distribution of velocities of $\mathrm{T}$-modes $\left(V_{\mathrm{T}}\right)$ in polycrystalline orientationally isotropic samples of elastically anisotropic cubic solid. In contrast to BLS peaks of L-modes, those of T-modes are difficult to measure due to a low scattering power as well as due to less favorable scattering geometries at which transverse modes can be detected. ${ }^{32}$ This could be one of the main reasons why high-pressure BLS measurements of $V_{\text {Tav }}$ at elevated pressures are not available for $\mathrm{NaCl}$, and those for solid argon resulted in $G(P)$ deviating by $100 \% .32,33$ Accordingly, discussion of a relation between the experimental $V_{\text {Tav }}$ data and the modelled-here $V_{\mathrm{T}}$-histograms and BLS peaks would be premature.

To obtain $V_{\mathrm{T}}$-histograms and to model BLS peaks of T-modes in polycrystalline $\mathrm{NaCl}$ and solid argon, we first calculated histograms for the slow $\left(V_{\mathrm{T} 1}\right)$ and fast $\left(V_{\mathrm{T} 2}\right)$ transverse modes in their single crystals and then summed them (Fig. 4). The calculations were performed at the same pressure conditions as above for the L-modes: For $\mathrm{NaCl}$, the $V_{\mathrm{T}}$ histograms were calculated at $P=1$ atm and $P=24 \mathrm{GPa}$. In the latter case the $C_{\mathrm{ij}}$ 's derived from our TDBS measurements were used. For solid argon, the $V_{\mathrm{T}}$ histograms were calculated at $P=2.4 \mathrm{GPa}$ and $P=49 \mathrm{GPa}$ using $C_{\mathrm{ij}}$ values reported elsewhere. ${ }^{30,9}$ To model the corresponding BLS peaks, the histograms were convoluted, as above, with Gaussian distributions having widths of 0.25 $\mathrm{GHz}$ and $1 \mathrm{GHz}$ (Fig. 4).

As can be recognized in Fig. 4, the histograms of T-modes spread over much wider $V_{\mathrm{T}}$ ranges (relative to the absolute $V_{\mathrm{T}}$ values) when compared with the corresponding $V_{\mathrm{L}}$ histograms in Fig. 3. Also, the $V_{\mathrm{T}}$ histograms exhibit two local maxima on different sides of the covered $V_{\mathrm{T}}$ range and the local maximum at the extreme $V_{\mathrm{T} 1\langle 100\rangle}=V_{\mathrm{T} 1\langle 110\rangle}=V_{\mathrm{T} 2\langle 100\rangle}$ is almost one order of magnitude higher than the second one near but not coinciding with $V_{\mathrm{T} 1\langle 111}=V_{\mathrm{T} 2\langle 111\rangle}$. In the case of $\mathrm{NaCl}$, the dominant maxima of the $V_{\mathrm{T}}$ histograms are located at $V_{\mathrm{Tmin}}$ and contain mostly contributions of the $V_{\mathrm{T} 1}$ modes (green lines in Fig. 4a, b). In the case of solid argon, the dominant maxima are located at $V_{\mathrm{T} \max }$ and contain mostly contributions of the $V_{\mathrm{T} 2}$ modes (blue lines in Fig. 4c, d). However, convolutions of the histograms with the Gaussian distributions (representing, respectively, an ideal and a typical instrument function of a BLS set-up) strongly suppress intensities of the dominant peaks: In the BLS signals modelled using the narrower Gaussian distribution with $\mathrm{FWHM}=0.25 \mathrm{GHz}$, the two local maxima can be 
recognized (gray solid curves in Fig. 4). Convolution with the wider Gaussian distribution obscured the two local maxima especially when the elastic anisotropy is not extremely high (black solid curves in Fig. 4), only the modelled BLS peaks become asymmetric. It seems to be very difficult to recognize these local maxima in real peaks of T-modes because they will be further obscured by noise, background and, eventually, sample texture. However, in some high-pressure experiments on polycrystalline samples of cubic $\mathrm{H}_{2} \mathrm{O}$ ice, splitting of BLS peaks of T-modes was reported. ${ }^{32}$ Authors of the work attributed the additional peak(s) to the polycrystalline nature of their samples without further specifications.
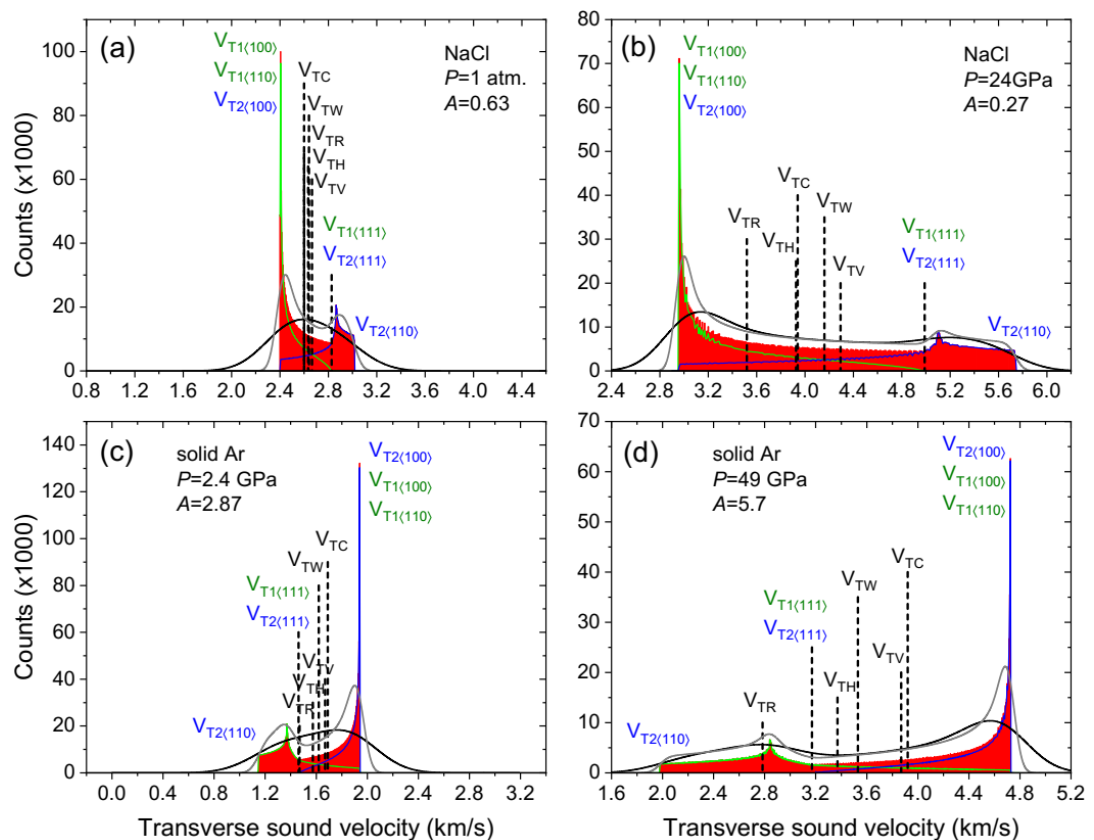

FIG. 4. Histograms of the frequency of occurrence of $V_{\mathrm{T}}$ values (comprising $V_{\mathrm{T} 1}$ and $V_{\mathrm{T} 2}$ ) in a cubic single crystal of $\mathrm{NaCl}(\mathrm{a}$ and $\mathrm{b}$ ) and in solid argon (c and d). The histograms were calculated using the same $C_{\mathrm{ij}}$ 's as by calculation of the $V_{\mathrm{L}}$ histograms in Fig. 3. Histograms for the slow $\left(V_{\mathrm{T} 1}\right)$ and fast $\left(V_{\mathrm{T} 2}\right)$ transverse modes are shown by green and blue solid lines, respectively. Their sums are shown by red bars. Vertical dashed lines indicate the velocities $V_{\mathrm{TV}}, V_{\mathrm{TR}}$ and $V_{\mathrm{TH}}$ obtained using the three well-known approximations, Voigt, Reuss, and Hill, respectively. Gray and black solid lines represent modelled shapes of BLS-peaks for isotropic densified polycrystalline samples. They were obtained via convolution of the histograms with Gaussian distributions having widths of $0.25 \mathrm{GHz}$ and of $1 \mathrm{GHz}$, respectively. The vertical dashed lines labeled with $V_{\mathrm{LC}}$ indicate positions of mass-centers of the histograms while $V_{\mathrm{LW}}$ indicate positions of FWHM-middles of the BLS peaks modelled using the wider Gaussian distribution.

Although the $V_{\mathrm{T}}$ distributions in Fig. 4 are very wide, especially when elastic anisotropy becomes pronounced (e.g. on pressure increase), the difference between $V_{\mathrm{TW}}$ and $V_{\mathrm{TH}}$ is less significant when compared with that between $V_{\mathrm{LW}}$ and $V_{\mathrm{LH}}$. Even though, $V_{\mathrm{TW}}$ shifts faster towards $V_{\mathrm{T} \text { max }}$ with increasing anisotropy than $V_{\mathrm{TH}}$, the overestimation of $V_{\mathrm{Tav}}=V_{\mathrm{TW}}$ recovered from a classical BLS measurement with respect to $V_{\mathrm{Tav}}=V_{\mathrm{TH}}$ measured using a PEUS technique appears to remain moderate (see also below). Finally, our results in Fig. 4 suggest that increase of elastic anisotropy is accompanied by a systematic shift of $V_{\mathrm{TW}}$ and $V_{\mathrm{TC}}$ to $V_{\text {Tmax }}$, independent of the anisotropy type, $A>1$ or $A<1$.

\section{Uncertainties in isotropic elastic moduli $B$ and $G$ due to elastic anisotropy}

As recognized above, elastic anisotropy can lead to a systematic overestimation of $V_{\text {Lav }}$ and $V_{\text {Tav }}$ recovered from BLS- and, in some cases, from PEUS measurements on dense orientationally isotropic polycrystalline samples even though the sample materials exhibit cubic structure. The deviation is systematic and can be predicted if $C_{\mathrm{ij}}$ values are known. The latter are, however, often not known for new materials not available or not accessible as single crystals. New compounds and phases forming at high pressures are just two examples 
of such materials. Below, we estimated deviations in determining of $B$ and $G$ when such overestimation of $V_{\mathrm{Lav}}$ and/or $V_{\mathrm{Tav}}$ takes place.

Firstly, we consider the frequent case when only one BLS peak of L-modes is detectable. Applying the equation relating $V_{\text {Lav }}$ in an isotropic polycrystalline solid to $B$ and $G$ :

$$
V_{\mathrm{Lav}}=\sqrt{\frac{3 B+4 G}{3 \rho}}
$$

and assuming that $B$ was determined correctly in an independent measurement, e.g. from an equation of state $\rho(P)$, we estimated the error for $G$ to be:

$$
\frac{\delta G}{G}=\left(\frac{3 B}{2 G}+2\right) \frac{\delta V_{\mathrm{Lav}}}{V_{\mathrm{Lav}}}
$$

To provide a quantitative example, we consider here the realistic differences in $V_{\text {Lav }}$ discussed above for solid argon at $P=49 \mathrm{GPa}$. Here, independent of the BLS peak quality, the operator will select, most probably, the value $V_{\mathrm{LW}} \approx V_{\mathrm{L}\langle 110)} \approx V_{\mathrm{LC}}$ as that corresponding to $V_{\mathrm{Lav}}$ while $V_{\mathrm{LH}}$ is $~ 7 \%$ lower. Applying the earlier published $B=190 \mathrm{GPa}^{31,33,35}$ and $G=57 \mathrm{GPa}^{9,33}$ at $P=49$ $\mathrm{GPa}$, we obtained the relative deviation of the shear modulus to be $\delta G / G \approx 50 \%$.

In the opposite case, when $G$ is correctly measured but $B$ is not known, the error in determining of $B$ is given by the equation:

$$
\frac{\delta B}{B}=\left(\frac{8 G}{3 B}+2\right) \frac{\delta V_{\mathrm{Lav}}}{V_{\mathrm{Lav}}}
$$

For the same case of solid argon at $P=49 \mathrm{GPa}$, the overestimation of $V_{\mathrm{Lav}}$ by the same $\sim 7 \%$ results in a deviation of the bulk modulus by $\delta B / B \approx 19.6 \%$. Thus, the overestimation caused by use of $V_{\text {Lav }}$ recovered from classical BLS measurements can be significant for $B$ but less dramatic than for $G$.

The second frequent case is a BLS spectrum showing two peaks due to L- and T-modes. In the case of orientationally isotropic polycrystalline sample of solid argon at $P=49 \mathrm{GPa}$, these peaks should have asymmetric shapes as shown in Fig. 3e and 4d, respectively. Applying the equation relating transverse sound velocity in a polycrystalline solid, $V_{\text {Tav }}$, and the isotropic shear modulus $G$ :

$$
V_{\mathrm{Tav}}=\sqrt{\frac{G}{\rho}}
$$

we obtained for solid argon at $P=49 \mathrm{GPa}$ the relative overestimation $\delta G / G=\left(V_{\mathrm{TW}}{ }^{2}-V_{\mathrm{TH}}{ }^{2}\right) / V_{\mathrm{TH}}{ }^{2}$ $=9.7 \%$. To estimate deviation of $B$ of solid argon, we used an equation similar to Eq. (11):

$$
\frac{\delta B}{B}=\left(\frac{8(G+\delta G)}{3 B}+2\right) \frac{\delta V_{\mathrm{Lav}}}{V_{\mathrm{Lav}}}
$$

and obtained almost the same $\delta B / B \approx 20.2 \%$ as above for single BLS peak of the L-modes. Summarizing, both $B$ and $G$ can be overestimated if classical BLS spectra collected from polycrystalline orientationally isotropic samples of elastically anisotropic cubic solids contain two peaks of the T- and L-modes. However, the overestimation of $G$ is much less dramatic than by use of the $V_{\text {Lav }}$ peak position in a BLS spectrum with a single L-peak and an independently measured $B$.

Finally, we consider uncertainty in the above systematic deviations of isotropic moduli if $C_{\mathrm{ij}}$ values from TDBS measurements are biased by an uncertainty in the used refractive index. As already mentioned in Section II, we used theoretical refractive index $n=1.698$ at $P=24 \mathrm{GPa}$ to derive the local $V_{\mathrm{L}}$ values in our polycrystalline $\mathrm{NaCl}$ sample. Here we apply, in addition, the earlier reported $n=1.68$ measured upon shock compression of $\mathrm{NaCl}^{36}$ in order to quantify influence of the $1 \%$-uncertainty in $n$ on $V_{\mathrm{L}^{-}}$and $C_{\mathrm{ij}}$ values of $\mathrm{NaCl}$ obtained from TDBS signals. For this aim, we used both $n$-values to derive first $V_{\mathrm{L}\langle 111\rangle}$ and $V_{\mathrm{L}\langle 100\rangle}$, then $C_{\mathrm{ij}}$ 's, and, finally, the values $V_{\mathrm{LH}}$ and $V_{\mathrm{LW}}$. Applying our theoretical $n=1.698$, we obtained $V_{\mathrm{LH}}=7.61$ $\mathrm{km} / \mathrm{s}, V_{\mathrm{LW}}=8.05 \mathrm{~km} / \mathrm{s}$ and the ratio $\left(V_{\mathrm{LW}}-V_{\mathrm{LH}}\right) / V_{\mathrm{LH}}=5.75 \%$. Applying the previously reported experimental $n=1.68$, we obtained $V_{\mathrm{LH}}=7.70 \mathrm{~km} / \mathrm{s}, V_{\mathrm{LW}}=8.11 \mathrm{~km} / \mathrm{s}$ and the ratio $\left(V_{\mathrm{LW}^{-}}\right.$ $V_{\mathrm{LH}} / V_{\mathrm{LH}}=5.32 \%$. For the value of interest, $\delta G / G$, we obtained, applying Eq. (10), 32.3\% and 
$28.7 \%$, respectively. Thus, the degree of elastic anisotropy derived from TDBS measurements is relatively sensitive to the used refractive index. However, our findings on systematic deviation of sound velocities and elastic moduli of polycrystalline samples of elastically anisotropic cubic solids, if derived from classical BLS and PEUS techniques, are not affected by the latter outcome in no way.

In classical BLS measurements, determining of $V_{\mathrm{Lav}}$ and $V_{\mathrm{Tav}}$ depends on the spectrum quality and its interpretation by the operator. ${ }^{10}$ Furthermore, in such measurements on microscopic samples compressed in a DAC, "averaged" sound velocity is sometimes derived from weak and noisy peaks overlapping with flanks of strong BLS-peaks of diamond anvils and, eventually, of a pressure transmitting medium. Background scattering and electronic noise make determining of $V_{\mathrm{Lav}}$ and $V_{\text {Tav }}$ even more demanding and counting times, for a single BLS spectrum, up to 72 hours are not rear. ${ }^{10,12}$ Even if all above considered aspects are taken into account and the experimental procedure optimized, sample texture can further bias the BLS-peak shapes and positions, or duration of propagation of acoustic pulses in PEUS measurements. It appears that, in the case of $\mathrm{NaCl}$, the much stronger approaching of $V_{\mathrm{L}\langle 111\rangle}$ by $V_{\text {Lav }}$ (see Fig. 2), when compared with that predicted by Eq. (3), is caused by a strong sample texture during the classical BLS- and PEUS measurements. ${ }^{26-28}$ Pronounced texturing of polycrystalline $\mathrm{NaCl}$ samples upon compression between opposed anvils was already documented. ${ }^{37}$

Some of the experimentalists who applied classical BLS to examine solids compressed in a DAC, including $\mathrm{NaCl}_{1}{ }^{10}$ considered various sources of experimental uncertainties and limitations of this technique. However, the systematic tendency of $V_{\mathrm{Lav}}(P)$ to approach $V_{\mathrm{L}\langle 111\rangle}(P)$, in the case of orientationally isotropic samples having cubic structures but exhibiting elastic anisotropy, was recognized and evaluated in the present work for the first time.

\section{Conclusion}

In this work, we extracted $V_{\mathrm{L}\langle 111\rangle}$ and $V_{\mathrm{L}\langle 100\rangle}$ in a single crystal of $\mathrm{NaCl}$ at $P=24 \mathrm{GPa}$ from the TDBS signals collected during propagation of narrow CAPs through a polycrystalline $\mathrm{NaCl}$ sample compressed in a DAC. Applying the Decker's EOS of $\mathrm{NaCl}$, considered as the reference for the primary pressure scale, we derived $C_{\mathrm{ij}}$ 's of $\mathrm{NaCl}$ at this pressure. We have confirmed the earlier recognized tendency of experimental $V_{\mathrm{Lav}}$ (for polycrystalline cubic solids with $A>1$ ) to approach $V_{\mathrm{L}\langle 111\rangle}$ but this time for a solid, $\mathrm{NaCl}$, exhibiting $A<1$. We have shown that this tendency is promoted by asymmetry of $V_{\mathrm{L}}$ distribution in a cubic single crystal because (i) the directions close to $\langle 110\rangle$ have the highest multiplicity and thus the strongest contribution to the BLS peak intensity and (ii) $V_{\mathrm{L}\langle 110\rangle}$ is always closer to $V_{\mathrm{L}\langle 111\rangle}$ than to $V_{\mathrm{L}\langle 100\rangle}$ independent of the anisotropy type, $A>1$ or $A<1$. For cubic solids with $A>1$, both BLS peak maximum and middle of the FWHM provide a higher $V_{\mathrm{Lav}}$ when compared with $V_{\mathrm{LH}}$. We have shown that influence of grain sizes, anisotropy type and degree, as well as of the method of signal analyses should be considered when $V_{\mathrm{Lav}}$ is derived from classical BLS- and PEUS measurements on polycrystalline elastically anisotropic cubic samples. If such $V_{\text {Lav }}$ is used alone to derive either the aggregate shear- of bulk modulus, then a moderate deviation of the $V_{\mathrm{Lav}}$ from $V_{\mathrm{LH}}$ can cause a strong error in elastic moduli while that in $G$ can be much greater than the error in $B$. A similar analysis of distribution of $V_{\mathrm{T}}$ in a cubic single crystal was also performed for solids having $A>1$ (solid argon) and $A<1(\mathrm{NaCl})$ and a much wider spreading of $V_{\mathrm{T}}$ velocities was revealed. Shapes of $V_{\mathrm{T}}$ histograms are different from those of $V_{\mathrm{L}}$ histograms but the characteristic features seem to be difficult to extract in real BLS measurements due to a low scattering intensity of T-modes, insufficient resolution and low signal-to-noise ratios of available experimental set-ups which cannot be compensated by long counting times. The latter explains, at least partially, why $V_{\operatorname{Tav}}(P)$ for the considered here solids, argon and $\mathrm{NaCl}$, are not available (the case for $\mathrm{NaCl}$ ) or are inconsistent (the case for solid argon). Another outcome of our analysis of the $V_{\mathrm{T}}$ distribution in cubic single crystals is a moderate difference between $G$ derived from $V_{\mathrm{Tav}}=V_{\mathrm{TW}}$ and $G_{\mathrm{H}}$, when compared with the case where the BLS peak of L-modes is used to derive $G$. Accordingly, errors in $B$ and $G$ obtained from classical BLS spectra containing both transverse- and longitudinal peaks are nearly independent and controlled by asymmetry of each of the peaks.

In contrast to BLS and PEUS, the applied here TDBS technique is much less sensitive to the elastic anisotropy sample material and its homogeneity: A large number of the depth- and 
laterally resolved measurements of local $V_{\mathrm{L}}$ values permits detecting, with a high degree of confidence, of the extremes $V_{\mathrm{L}\langle 111\rangle}$ and $V_{\mathrm{L}\langle 100\rangle}$ even when single crystals are not accessible and the examined polycrystalline sample is textured. If combined with independently measured bulk modulus and density, e.g. from an EOS, a complete set of $C_{\mathrm{ij}}$ and thus the true $V_{\mathrm{Lav}}=V_{\mathrm{LH}}$ and $V_{\mathrm{Tav}}=V_{\mathrm{TH}}$ can be determined for any solid exhibiting cubic structure.

\section{Acknowledgements}

The authors acknowledge financial support from the NSFC (projects No. 41504070 and No. 41874103), the CSC (File No. 201606955092), and the French National Research Agency, ANR (project I2T2M, No. ANR-18-CE42-0017). We thank E. Peronne for experimental assistance, L. Becerra for sample preparation, X. Liu and Y. Wu for discussions.

\section{Data Availability}

The data that support the findings of this study are available within the article. The raw TDBS signals can be provided on request by $\mathrm{F}$. Xu.

\section{References:}

1 W. Voigt, Lehrbuch der Kristallphysik. Leipzig, Teubner (1928).

2 R. Hill, Proc. Phys. Soc. A 65, 349 (1952).

3 Z. Hashin and S. Shtrikman, J. Mech. Phys. Solids 10, 335 (1962).

${ }^{4}$ Z. Hashin and S. Shtrikman, J. Mech. Phys. Solids 10: 343 (1962).

5 J. M. Brown, Comput. Geosci. 80, 95 (2015).

6 C. S. Zha, H. K. Mao, and R. J Hemley, P. Natl. Acad. Sci. USA 97, 13494 (2000).

7 S. R. Shieh, T. S. Duffy, and G. Shen, Phys. Earth Planet In. 143, 93 (2004).

8 M. Kuriakose, S. Raetz, Q. M. Hu, S. M. Nikitin, N. Chigarev, V. Tournat, A. Bulou, A. Lomonosov, P. Djemia, V. E. Gusev, and A. Zerr, Phys. Rev. B 96, 134122 (2017).

9 S. Raetz, M. Kuriakose, P. Djemia, S. M. Nikitin, N. Chigarev, V. Tournat, A. Bulou, A. Lomonosov, V. E. Gusev, and A. Zerr, Phys. Rev. B 99, 224102 (2019).

${ }^{10}$ S. Sinogeikin, J. Bass, V. Prakapenka, D. Lakshtanov, G. Shen, C. Sanchez-Valle, and M. Rivers, Rev. Sci. Instrum. 77, 103905 (2006).

${ }^{11}$ J. Wang, J. Li, S. Yip, S. Phillpot, and D. Wolf, Phys. Rev. B 52, 12627 (1995).

${ }^{12}$ M. Murakami, Y. Ohishi, N. Hirao, and K. Hirose, Earth Planet. Sci. Lett. 277, 123 (2009).

${ }^{13}$ F. Decremps, L. Belliard, B. Perrin, and M. Gauthier, Phys. Rev. Lett. 100, 035502 (2008).

${ }^{14}$ S. M. Nikitin, N. Chigarev, V. Tournat, A. Bulou, D. Gasteau, B. Castagnede, A. Zerr, and V. E. Gusev, Sci. Rep. 5, 9352 (2015).

${ }^{15}$ M. Kuriakose, S. Raetz, N. Chigarev, S. M. Nikitin, A. Bulou, D. Gasteau, V. Tournat, B. Castagnede, A. Zerr, and V. E. Gusev, Ultrasonics 69, 259 (2016).

${ }^{16}$ Z. Y. Mi, Ph.D. thesis, The University of Western Ontario (2013).

${ }^{17}$ S. Sandeep, T. Threard, E. De Lima Savi, N. Chigarev, A. Bulou, V. Tournat, A. Zerr, V. Gusev, and S. Raetz, arXiv:2008.00034 (2020).

${ }^{18}$ F. Xu, L. Belliard, D. Fournier, E. Charron, J.-Y. Duquesne, S. Martin, C. Secouard, and B. Perrin, Thin Solid Films 548, 366 (2013).

${ }^{19}$ F. Xu, Y. Guillet, S. Ravaine, and B. Audoin, Phys. Rev. B 97, 165412 (2018).

${ }^{20}$ B. Zhao, F. Xu, L. Belliard, H. J. Huang, B. Perrin, P. Djemia, and A. Zerr, Phys. Status Solidi RRL 13, 1900173 (2019).

${ }^{21}$ F. Xu, L. Belliard, C. Li, P. Djemia, L. Beccerra, H. Huang, B. Perrin, and A. Zerr, "Single-crystal elastic moduli, anisotropy and the B1-B2 phase transition of $\mathrm{NaCl}$ at high pressures" (to be submitted). 
${ }^{22}$ D. L. Decker, J. Appl. Phys. 42, 3239 (1971).

${ }^{23}$ A. E. H. Love, A Treatise on the Mathematical Theory of Elasticity (Dover, New York, 1944).

${ }^{24}$ C. H. Whitfield, E. M. Brody, and W. A. Bassett, Rev. Sci. Instrum. 47, 942 (1976).

${ }^{25}$ H. Kinoshita, N. Hamaya, and H. Fujisawa, J. Phys. Earth 27, 337 (1979).

${ }^{26}$ A. J. Campbell and D. L. Heinz, Science 257, 66 (1992).

${ }^{27}$ J. Frankel, F. J. Rich, and C. G. Homan, J. Geophys. Res. 81, 6357 (1976).

${ }^{28}$ M. Matsui, Y. Higo, Y. Okamoto, T. Irifune, and K. I. Funakoshi, Am. Mineral. 97, 1670 (2012).

${ }^{29}$ W. A. Bassett, T. Takahashi, H. K. Mao, and J. S. Weaver, J. Appl. Phys. 39, 319 (1968).

${ }^{30}$ H. Shimizu, H. Tashiro, T. Kume, and S. Sasaki, Phys. Rev. Lett. 86, 4568 (2001).

${ }^{31}$ P. Richet, J.-A. Xu, and H. K. Mao, Phys. Chem. Minerals 16, 207 (1988).

${ }^{32}$ B. Chen, A. E. Gleason, J. Y. Yan, K. J. Koski, S. Clark, and R. Jeanloz, Phys. Rev. B 81, 144110 (2010).

${ }^{33}$ H. Marquardt, S. Speziale, A. Gleason, S. Sinogeikin, I. Kantor, and V. B. Prakapenka, J. Appl. Phys. 114, 093517 (2013).

${ }^{34}$ M. Ahart, M. Somayazulu, S. A. Gramsch, R. Boehler, H.-K. Mao, and R. J. Hemley, J. Chem. Phys. 134, 124517 (2011).

${ }^{35}$ D. Errandonea, R. Boehler, S. Japel, M. Mezouar, and L. R. Benedetti, Phys. Rev. B 73, 092106 (2006).

${ }^{36}$ J. Wackerle and H. L. Stacy, Shock Waves in Condensed Matter, 699 (1987).

${ }^{37}$ Z. Mi, S. R. Shieh, A. Kavner, B. Kiefer, H. R. Wenk, and T. S. Duffy, J. Appl. Phys. 123, 135901 (2018). 


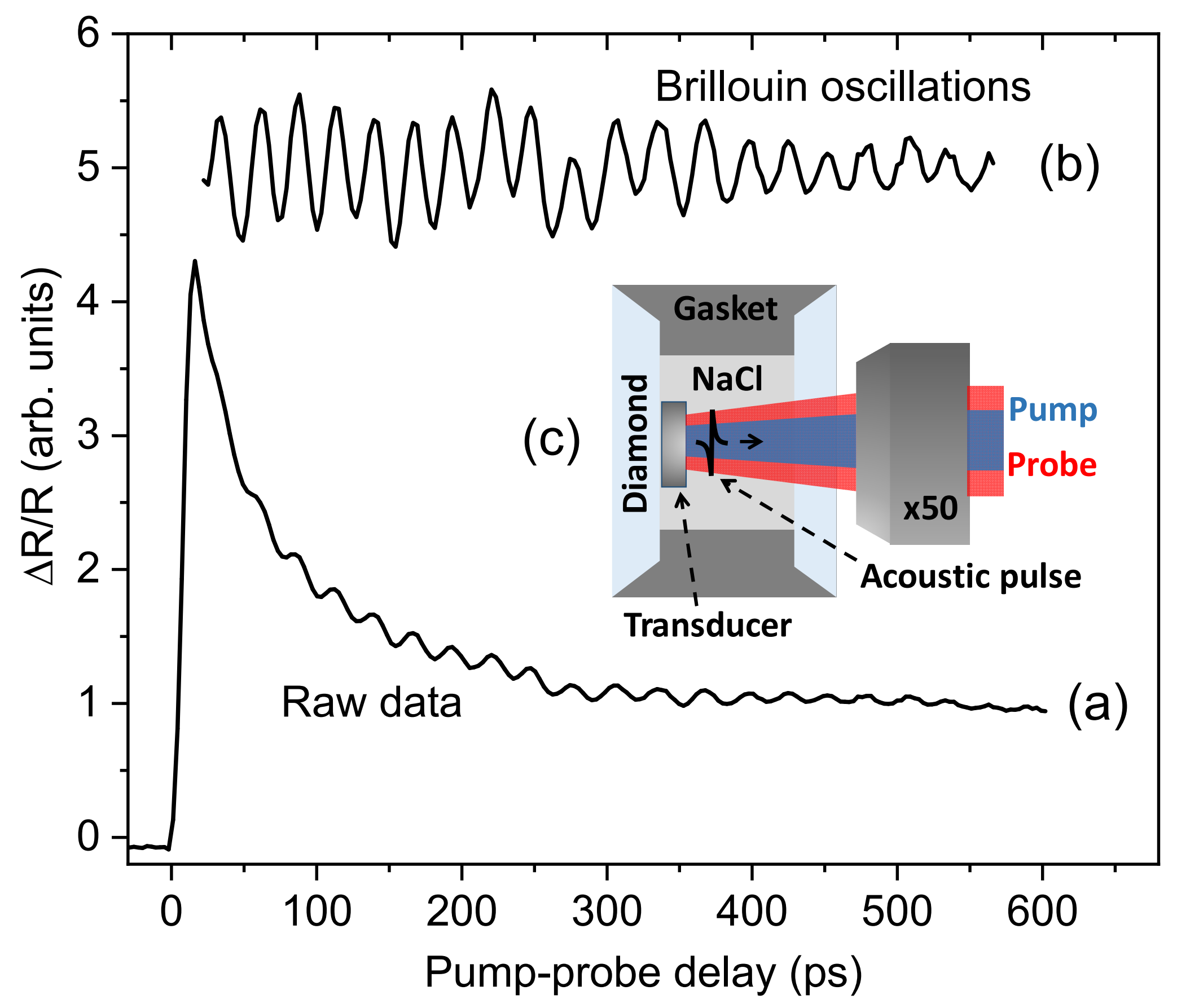




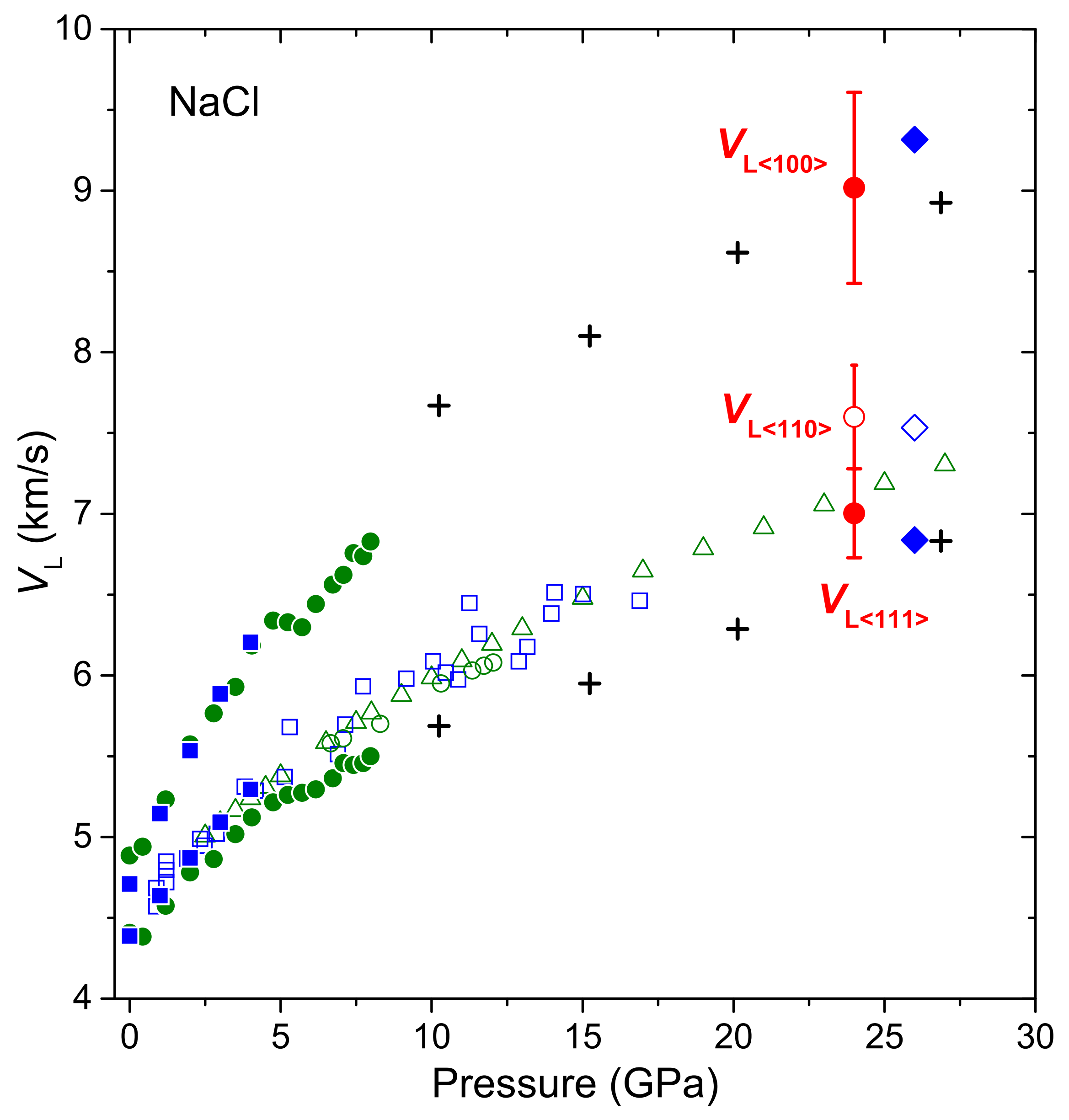



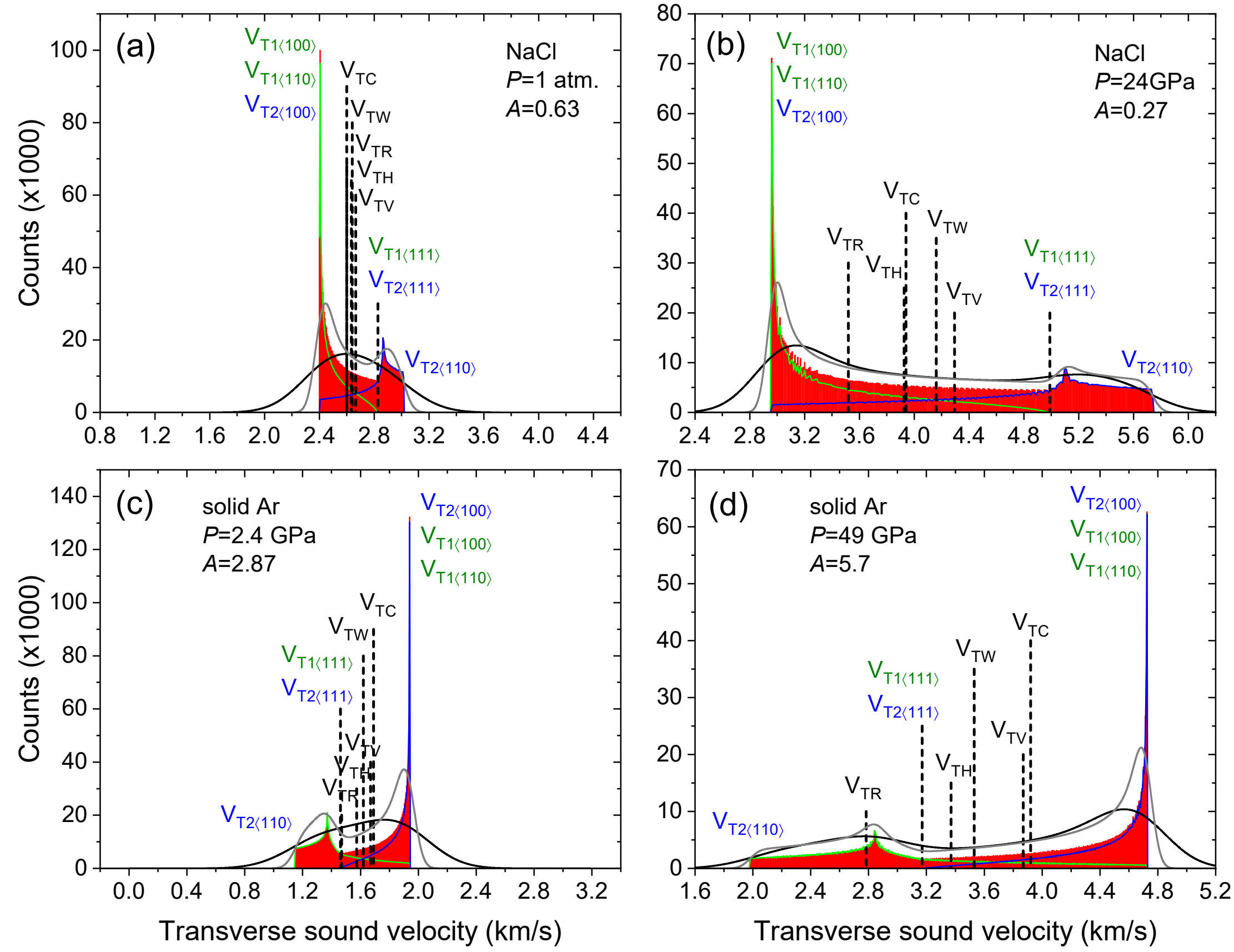